\title{
A modified embedded atom method potential for interstitial oxygen in titanium
}

\author{
Pinchao Zhang, Dallas R. Trinkle* \\ Department of Materials Science and Engineering, University of Illinois, Urbana-Champaign
}

\begin{abstract}
Modeling oxygen interstitials in titanium requires a new empirical potential. We optimize potential parameters using a fitting database of first-principle oxygen interstitial energies and forces. A new database optimization algorithm based on Bayesian sampling is applied to obtain an optimal potential for a specific testing set of density functional data. A parallel genetic algorithm minimizes the sum of logistic function evaluations of the testing set predictions. We test the transferability of the potential model against oxygen interstitials in HCP titanium, transition barriers between oxygen interstitial sites, and oxygen in the titanium prismatic stacking fault. The potential predicts that the interaction between oxygen and a screw dislocation core is weak and short-ranged.

Keywords: classical potential, point defects, diffusion, dislocations
\end{abstract}

\section{Introduction}

Titanium alloys have good strength-to-weight ratios, high toughness, high melting temperature, and excellent corrosion resistance, which make them promising candidates for many structural applications especially in aerospace and aviation.[1] The introduction of $\mathrm{O}$ in Ti increases the yield strength but reduces the ductility.[2, 3] In-situ transmission electron microscopy compression tests on Ti nanopillars with different $\mathrm{O}$ concentrations suggest that the strength increase comes from an interaction between

\footnotetext{
* Corresponding author

Email address: dtrinkle@illinois. edu (Dallas R. Trinkle)
}

Preprint submitted to Journal of Computational Materials Science

July 26, 2016

(C) 2016. This manuscript version is made available under the Elsevier user license http://www.elsevier.com/open-access/userlicense/1.0/ 
the $\mathrm{O}$ interstitial atoms and the screw dislocation core.[4] Previous experiments along with crystallographic models also show that oxygen interstitials impede (1012) Ti twin growth, indicating that $\mathrm{O}$ could affect the deformation properties of Ti. [5, 6] $\mathrm{Wu}$ and Trinkle used density functional theory (DFT) to calculate oxygen diffusion in HCP $\mathrm{Ti}$, where they found a new stable crowdion site-in addition to the known octahedral and hexahedral sites - that also contributes to $\mathrm{O}$ diffusion in HCP Ti.[7] Moreover, Ghazisaeidi and Trinkle DFT calculations of O interaction with the (1012) Ti twin boundary and the (1010) prismatic stacking fault showed that some oxygen interstitial sites at the twin boundary are attractive while others are repulsive, and that oxygen increases the prismatic stacking fault energy.[8] DFT also predicts that oxygen will decrease the Ti basal stacking fault energy.[9] Efficient and accurate empirical potentials that capture all the Ti-O interaction effects are not present, which is essential for large molecular dynamics simulations of oxygen interaction with "large" Ti defects such as dislocations, grain and twin boundaries, crack tips, and other defects that are difficult to simulate from first-principles.

Modified embedded-atom method (MEAM) potentials have been widely used to predict structural properties for metals. Baskes[10, 11] extended the embedded atom method[12, 13] to include the angular dependent three-body term in the electron density. Joost et al. recently developed an analytic MEAM potential to model Ti oxides; however, the potential was not optimize to modeling $\mathrm{O}$ interaction with defects in Ti.[14] Spline-based MEAM potentials offer enhanced flexibility and efficiency over analytic MEAM potentials.[15] Three successful single-element implementations on $\mathrm{Si}[15], \mathrm{Ti}[16]$ and $\mathrm{Mo}[17]$ have been published, but multicomponent MEAM models are still difficult to optimize. For our situation, the spline-based Ti MEAM potential developed by Hennig et al. [16] to study the martensitic phase transition between $\alpha, \beta$ and $\omega$ Ti phases also predicts a similar Ti screw dislocation core structure as DFT.[18] We build on this model to include Ti-O interactions within MEAM formalism to study $\mathrm{O}$ near dislocations in Ti.

We start with the development of the binary MEAM potential functional form. The Ti MEAM potential[16] parameters are unchanged for Ti-Ti interactions, and we introduce Ti-O interactions. We construct a DFT database of structural energies and forces, 
and use the force-matching method[19] to optimize oxygen interaction functions. We expand the potential fitting problem to the optimization of the fitting database itself, by using an objective function based on testing errors, and optimized using our new database optimization algorithm.[20] The objective function consists of quantitative assessments of the testing set predictions of the potential model given by the logistic function. A parallel genetic algorithm minimizes the objective function and provides optimal weights for each entry in the fitting database. We test the transferability of the Ti-O potential for $\mathrm{O}$ interstitial sites in HCP Ti, transition barriers between the interstitial sites, and $\mathrm{O}$ in the Ti prismatic stacking fault. Finally, we use our Ti-O potential to study $\mathrm{O}$ interacting with a Ti screw dislocation: exploring the stable sites near the dislocation core, and the oxygen-induced changes in the Ti dislocation core structure.

\section{Optimization of the Binary MEAM potential for Titanium-Oxygen}

\subsection{Potential functional form}

We develop a spline-based binary MEAM potential for Ti-O. The MEAM potential functional form is[15],

$$
E=\sum_{i<j} \phi_{i j}\left(r_{i j}\right)+\sum_{i} U_{i}\left(n_{i}\right)
$$

where $\phi_{i j}(r)$ is the pair interaction between atoms $i$ and $j, U_{i}\left(n_{i}\right)$ is the embedding energy, and the density $n_{i}$ at atom $i$ is,

$$
n_{i}=\sum_{j \neq i} \rho_{j}\left(r_{i j}\right)+\sum_{\substack{j<k, j, k \neq i}} f_{j}\left(r_{i j}\right) f_{k}\left(r_{i k}\right) g_{j k}\left(\cos \theta_{j i k}\right)
$$

The indices $i$ and $j$ range over all atoms in the system. The angle $\theta_{j i k}$ is the bond angle between atoms $j, i$, and $k$, centered on $i$. We employ the idea from the embedded atom method, where the density of the impurity atom $i$ only depends on the other "host" atoms. Therefore, the functions $\rho, f$ and $g$ are selected based on the neighbors of $i$, but do not depend on the chemical identity of atom $i$. We use the Ti MEAM potential[16] for Ti-Ti interactions and neglect $\mathrm{O}-\mathrm{O}$ interactions due to the dilute limit assumption. We fit the cubic spline functions for the Ti-O pair interaction 
$\phi_{\mathrm{TiO}}(r)$, the $\mathrm{O}$ density function $\rho_{\mathrm{O}}(r)$, the $\mathrm{O}$ embedding function $U_{\mathrm{O}}(n)$, and the functions $f_{\mathrm{O}}(r)$ and $g_{\mathrm{TiO}}(\cos \theta)$ which comprise the three-body contributions to the density $n_{i}$. The MEAM potential calculations are done using the molecular dynamics code LAMMPS. 21]

Our new database optimization algorithm[20] offers a quantitative and automated way of optimizing an empirical potential. A conventional potential fitting process uses weighted least-squares minimization to find the best set of parameters with numerous manual trial and error attempts to "tune" the weights appearing in the squared error. Multiple local minima of the squared errors can also make the development of empirical potential model time-consuming and heavily guided by individual perception. We formulate the optimization of the empirical potential model into a two-step optimization problem. First, we minimize the weighted least-squared error against a fitting database to optimize the potential parameters. We can also define an objective function for a fitting database, with its optimal potential parameters based on errors against a larger testing set to measure the "transferability" of the potential model. Second, we optimize the fitting database by minimizing the objective function to find the optimal weight values as the input of the next iteration. This two step process repeats in a cycle to find the best potential parameters and fitting database that produces those parameters. Following Ref. [20], we use a Bayesian statistical framework with weighed least-squares fitting; the likelihood of the potential parameters $\theta$ for a given fitting database $F$,

$$
L(F \mid \theta) \propto \exp \left(-\frac{1}{W} \sum_{\alpha \in F} w_{\alpha} \epsilon_{\alpha}^{2}(\theta)\right),
$$

where $w_{\alpha}$ is the relative weight for entry $\alpha$ in the fitting database, $W=S\left(\theta^{\mathrm{MLE}}, F\right)$, and $\theta^{\mathrm{MLE}}$ is the maximum likelihood estimation (MLE) of the likelihood function. The objective function of a fitting database $F$ given a testing set $T$ (consisting of structural energies, forces, or other potential predictions) is,

$$
O(F \mid T)=\sum_{\beta \in T} C\left(\left\langle\epsilon_{\beta}^{2}(\theta)\right\rangle_{F}, \epsilon_{\beta 0}\right),
$$

where the $C\left(x, \epsilon_{0}\right)$ is the cost function of a prediction of the potential for ensemble average error $\left\langle\epsilon_{\beta}^{2}(\theta)\right\rangle_{F}$ and "decision boundary" value $\epsilon_{\beta 0}$ (see below). The ensemble 
average $\left\langle\epsilon_{\beta}^{2}(\theta)\right\rangle_{F}$ is calculated using Bayesian sampling in the neighboring domain of the $\theta^{\mathrm{MLE}}$ to account for the MLE errors and the flexibility of the model under small variations in parameters[22], the so-called "sloppiness" of the model.

We choose the logistic function [23],

$$
C\left(x, \epsilon_{0}\right)=\frac{1}{1+\exp \left(-m\left(\left(x / \epsilon_{0}\right)-1\right)\right)},
$$

for our cost function as it mimics the evaluation process of predictions for a classical potential. We wish to balance errors, where the ideal outcome is all the testing set errors are below acceptable thresholds. Once a prediction falls below the threshold, decreasing the error of the same entry does not significantly improve the transferability of the potential. On the other hand, if a prediction error is very large, making worse predictions does not affect the transferability of the potential (with obvious caveats about the introduction of artificially low energy structures). The logistic function captures these two key features in potential fitting, which makes it a good candidate for the objective function. The derivative of the logistic function is not zero, which maintains the improvement by error reduction compared to a step function. The smooth functional form is also straightforward to calculate and couple with optimization algorithms such as gradient descent and genetic algorithms[24] (GA). The stiffness of the logistic function is determined by $m$. As $m \rightarrow \infty$, the logistic function becomes the step function. We identify $\epsilon_{0}$ as the "decision boundary" in classification regression analysis, which determines the "acceptable" error threshold for each entry in the testing set. The decision boundary for the energy entries and force entries in this work are $0.2 \mathrm{eV}$ and $0.5 \mathrm{eV} / \AA \AA$, respectively. The energy error boundary denotes the threshold of the error in total energy differences between two structures. The force error boundary denotes the threshold for the root of sum of squared errors for all force components on all atoms in a structure. We represent the optimal fitting database as the optimal weight set and the set of parameters with the least squared-errors in the fitting database. The decision boundaries are chosen to produce errors of less than $0.2 \mathrm{eV}$ in activation barriers for diffusion (c.f.Table 4). 


\subsection{Ab initio Database and Optimization Algorithm}

We perform density functional theory (DFT) calculations using the planewave basis code VASP [25, 26] for various Ti-O configurations to build the fitting database and the testing set for the potential fitting process[27]. The fitting database consists of DFT energies and forces for $\mathrm{O}$ in $\mathrm{HCP}$ Ti at the octahedral (o), hexahedral (h) and crowdion (c) interstitial sites, and for the transition state configurations, oh, oc, hc and oo, between these sites evaluated using the nudged-elastic band method (NEB).[28] While these geometries are relaxed in DFT, they will not be relaxed during the evaluation of errors for the likelihood function; instead, the energies and forces will be evaluated, and the target value for the forces will be zero. The testing set includes the fitting database structures as well as configurations with $\mathrm{O}$ displaced from interstitial sites by a fixed distance $(0.1 \AA$ and $0.05 \AA$ ) in random directions, and prismatic stacking fault configurations with $\mathrm{O}$ in octahedral site of Ti fault plane (1010). In the case of the testing set error evaluation, the three DFT relaxed interstitial site geometries are relaxed with the classical potential before evaluating the energy differences predictions. The supercells for $\mathrm{O}$ interstitial sites contain one $\mathrm{O}$ atom and $96 \mathrm{Ti}$ atoms $(4 \times 4 \times 3)$, and the stacking fault configuration contains one $\mathrm{O}$ atom and $80 \mathrm{Ti}$ atoms $(10 \times 2 \times 2)$. We treat $\mathrm{Ti}$ and $\mathrm{O}$ using Vanderbilt-type ultrasoft pseudopotentials [29, 30], with electronic configurations $[\mathrm{Ar}] 3 d^{3} 4 s^{1}$ and $[\mathrm{He}] 2 s^{2} 2 p^{4}$, respectively. A k-point mesh of $2 \times 2 \times 2$ with Methfessel-Paxton smearing of $0.2 \mathrm{eV}$ and plane-wave cutoff of $400 \mathrm{eV}$ is chosen so that the energies are converged to less than $1 \mathrm{meV} /$ atom. 31]

We use Bayesian sampling to generate an ensemble of parameters to evaluate the objective function.[20] We use a Markov-chain Monte Carlo algorithm, initiated from the maximum likelihood parameter estimate to calculate the mean squared errors for testing set entries. The potential ensemble has $5 \times 10^{3}$ independent parameters, from a total of $10^{6}$ steps. The acceptance ratios for the Monte Carlo simulations range from 0.25 to 0.6 and the autocorrelation length is about 200 steps. Every Monte Carlo step includes LAMMPS energy and force calculations for all the structures in the fitting database. In total, we perform $10^{9}$ LAMMPS calculations over the course of 1000 steps in our GA optimization.

We use an embarrassingly-parallel genetic algorithm to find an optimal fitting database. 
We first parallelize over children (weight sets in the fitting database) in the genetic algorithm, and then further distribute over the structures in the fitting database for each GA child. Random weights are initialized for the first generation. New fitting databases are made through a evolution process via three operations: selection, crossover and mutation. [24] We combine with gradient descent algorithm to find the local minimum of each set of weights to accelerate the convergence using the potential ensemble.[20] We maintain a set of 10 best chromosomes that GA finds during the optimization. The iteration continues until the stopping criterion is met: maximum number of GA iterations (1000) or the best set of chromosomes remain unchanged for over 100 steps. We select the optimal database from a set of databases with low objective function values, and find the fitting database that provides the best structural property predictions.

\section{Optimal Ti-O potential}

Table 1 lists the optimal weights in the fitting database and the logistic objective function values of the corresponding entries in the testing set. We scale the force errors to be $1 / \sqrt{10}$ so that the energy and force entries have similar error magnitudes, which makes it possible to compare the weights of energy and force entries in the database. None of the entries originally proposed in the fitting database have optimal weights of zero; hence, each is providing non-overlapping information to optimize the potential. The interstitial energy differences have the highest weights among all the entries. The high weight for the force entry of the h-site improves the characterization of the hexahedral atomic environment, while low weight for the c-site force entry reflects larger errors in the relaxed energy difference between the c- and o-site. For energy barriers of transitions from the c-site, large force errors for the two transition states lead to further differences in the transition state energies. On the other hand, low force errors for the transition state of o-o can compensate the errors for the o-o energy entry and result in a reasonable transition state energy estimation.

Figure 1 and Table 2 present the fitted cubic spline functions and the optimal potential parameters. Unlike the Ti-Ti MEAM potential, we use natural boundary conditions (zero second derivatives) at the inner cutoff for the radial functions, embedding func- 
Table 1: Genetic algorithm optimal weights and logistic values for the fitting database entries. The sum of the weights is one. The logistic value for the energies is based on a decision boundary of $0.2 \mathrm{eV}$, and the $0.5 \mathrm{eV} / \AA \AA$ for the forces. The energy entries for interstitial energy differences have the largest weights. The energy entries have lower logistic value than the forces. The o-o energy entry has the largest errors. The o-site and o-o state have the smallest force errors.

\begin{tabular}{cccccc}
\hline \hline Entry & weights & Logistic & Entry & weights & Logistic \\
\hline$E_{\mathrm{h}}-E_{\mathrm{o}}$ & 0.283 & 0.314 & $F_{\mathrm{o}}$ & 0.0682 & 0.297 \\
$E_{\mathrm{c}}-E_{\mathrm{o}}$ & 0.152 & 0.576 & $F_{\mathrm{h}}$ & 0.0300 & 0.632 \\
$E_{\mathrm{oh}}-E_{\mathrm{o}}$ & 0.0101 & 0.465 & $F_{\mathrm{c}}$ & 0.00362 & 0.546 \\
$E_{\mathrm{oc}}-E_{\mathrm{o}}$ & 0.0948 & 0.172 & $F_{\mathrm{oh}}$ & 0.0460 & 0.698 \\
$E_{\mathrm{hc}}-E_{\mathrm{h}}$ & 0.0898 & 0.171 & $F_{\mathrm{oc}}$ & 0.07665 & 0.533 \\
$E_{\mathrm{oo}}-E_{\mathrm{o}}$ & 0.0689 & 0.789 & $F_{\mathrm{hc}}$ & 0.0372 & 0.603 \\
& & & $F_{\mathrm{oo}}$ & 0.0395 & 0.352 \\
\hline \hline
\end{tabular}

tion and angular-dependent function. We choose the same outer cutoff, $5.5 \AA$, for all radial functions, $\phi(r), \rho(r)$ and $f(r)$. The inner cutoff is $1.9 \AA$, which is the smallest Ti-O distance among the seven structures in the fitting database. The pair potential $\phi(r)$ has a repulsive interaction for Ti-O distance from 2.5 Åto 4.1 Åand attractive interaction from 4.1 Åto the cutoff distance $5.5 \AA$ A. The Ti-O interaction for Ti-O distances close to the inner cutoff is attractive, which could be due to the lack of short Ti-O distance data in the fitting database. The density domain of the $\mathrm{O}$ embedding function is based on the Ti density at the interstitial sites in the seven structures. The energy landscape of the seven structures agrees with the embedding function energy contribution of the $\mathrm{O}$ atom. This indicates that the GA potential is able to reproduce the potential energy space accurately for Ti-O interaction.

\section{Tests for the Ti-O potentials}

We test the optimal fitting database by considering the force error distribution from the sampled distribution of potential parameters. We also test the optimal potential's predictions of energy barriers, stacking fault energies with oxygen, and the oxygen 

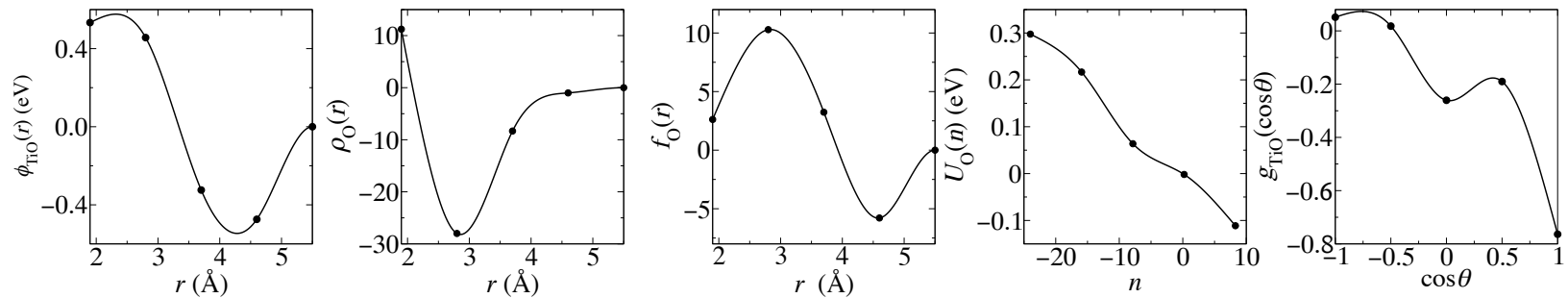

Figure 1: The five cubic spline functions for the $\mathrm{O}$ related functions in the GA optimized Ti-O MEAM potential. The radial functions $\phi_{\mathrm{TiO}}(r), \rho_{\mathrm{O}}(r)$ and $f_{\mathrm{O}}(r)$ have fixed boundary conditions at the cutoff of $5.5 \AA$, where the spline knot values and first derivatives are zero. The rest of the boundary conditions are natural boundary conditions, where the second derivatives of the spline functions are zero. The inner cutoffs of the radial spline functions match the lowest Ti-O distance present in the fitting database. The domain of the embedding function is located between the maximum and minimum of the $\mathrm{O}$ densities in the seven fitting database structures.

Table 2: Potential parameters of five cubic spline functions from GA optimization of the fitting database. Knot positions are equally spaced, while the function values at the knot points were treated as fitting parameters. The top table has the radial spline functions, $\phi_{\mathrm{TiO}}(r), \rho_{\mathrm{O}}(r)$ and $f_{O}(r)$, and the bottom table the embedding function, $U_{\mathrm{O}}(n)$ and angular dependent function, $g_{\mathrm{TiO}}(\cos \theta)$.

\begin{tabular}{ccccc}
\hline \hline$i$ & $r_{i}(\AA)$ & $\phi_{\mathrm{TiO}}(r)(\mathrm{eV})$ & $\rho_{\mathrm{O}}(r)$ & $f_{\mathrm{O}}(r)$ \\
\hline 0 & 1.900 & 0.533321679606674 & 11.2293641315584 & 2.62105440156724 \\
1 & 2.800 & 0.456402081843862 & -27.9976343076148 & 10.2850803058354 \\
2 & 3.700 & -0.324281383502201 & -8.32979773113248 & 3.23933763743897 \\
3 & 4.600 & -0.474029826906675 & -1.00863195297399 & -5.79049355858613 \\
4 & 5.500 & 0 & 0 & 0 \\
\hline \hline$i$ & $n_{i}$ & $U_{\mathrm{O}}(\rho)(\mathrm{eV})$ & $\cos \theta$ & $g_{\mathrm{TiO}}(\cos \theta)$ \\
\hline 0 & -23.993 & 0.297607384684645 & -1.000 & 0.0513843442016519 \\
1 & -15.924 & 0.216691597077105 & -0.500 & 0.0179024412245673 \\
2 & -7.855 & 0.0637598673719069 & 0.000 & -0.260650876879273 \\
3 & 0.213 & -0.00183450621970427 & 0.500 & -0.190163791764901 \\
4 & 8.282 & -0.111277018874367 & 1.000 & -0.763795416646599 \\
\hline \hline
\end{tabular}


interaction with a titanium screw dislocation.

Figure 2 shows that the force errors for nearest-neighbor (NN) Ti atoms of the $\mathrm{O}$ atom in the octahedral site are smaller than those of hexahedral and crowdion sites. Since the DFT forces on the atoms of the three relaxed interstitial geometries are zero, the force errors are the forces from a potential in the likelihood sample. The optimal weight value in Table 1 for the octahedral site forces is twice as large as the hexahedral and an order of magnitude larger than the crowdion. The force error distribution of the first NN of all three site has noticeable bias along the Ti-O directions, which shows the limitation of the potential when the distance between $\mathrm{O}$ and $\mathrm{Ti}$ are small. The force errors decrease as the $\mathrm{Ti}$ gets further away from the $\mathrm{O}$ atom. The hexahedral has the largest first $\mathrm{NN}$ force error, followed by the octahedral, then the crowdion with the smallest; this follows the increasing first neighbor distance of $1.92 \AA, 2.00$ Aand 2.09 $\AA$ A. However, the second NN for the octahedral site results in overall low force errors. In comparison, the second $\mathrm{NN}$ to the h-site has smaller force errors than the second $\mathrm{NN}$ atom compared to the c-site, as the database produces potentials that describe the atomic environment of the h-site more accurately than that of the c-site. The third NN distances for the three structures are $3.89 \AA$, $3.42 \AA$ and $3.44 \AA$, respectively. The o-site and h-site have small bias along the Ti-O direction for the third NN Ti atom, whereas the errors of the c-site are both the largest and are distributed along the Ti-O direction. Hence, the c-site has the least accurate atomic environment description among the three, and the octahedral site has the smallest errors. The force error distribution is a projection of the relaxed interstitial energy predictions, which the potential may have better predictions for hexahedral energy level than the crowdion energy level.

Table 4 demonstrates the transferability of the potential to the relaxed interstitial site energy levels and the transition barriers, which are not in the fitting database. For the relaxed interstitial energy levels, both our potential and the analytic Ti-O potential[14] predict the hexahedral site energy accurately. We attribute the accuracy to large weight contributions in the fitting database for the unrelaxed energy difference of o and h. Our potential underestimates the crowdion energy level due to large crowdion force errors. However, our ensemble includes the DFT value in the distribution of high likelihood potentials (within one standard deviation of the mean). For the NEB transition barrier 
$1 \mathrm{NN}$

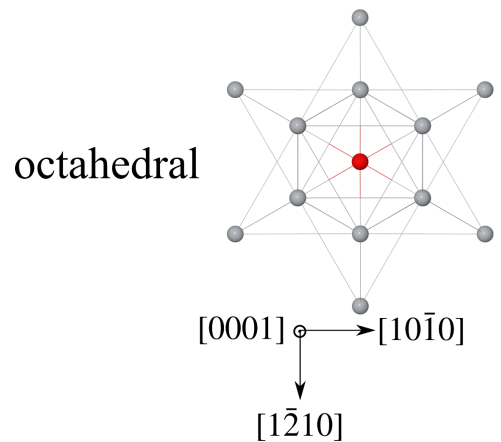

hexahedral

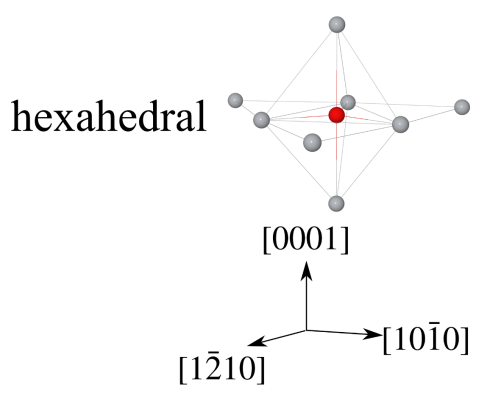

0

crowdion
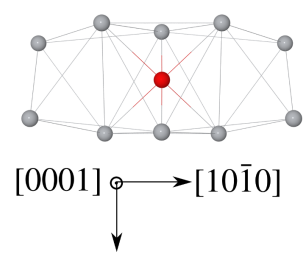

$[1 \overline{2} 10]$
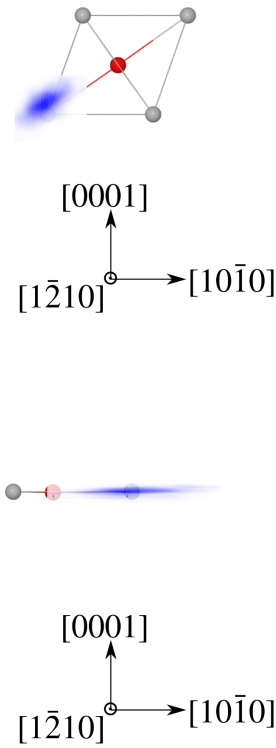

○

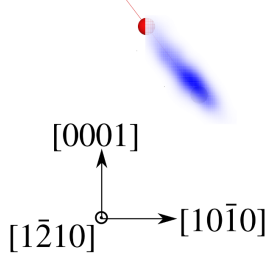

$2 \mathrm{NN}$
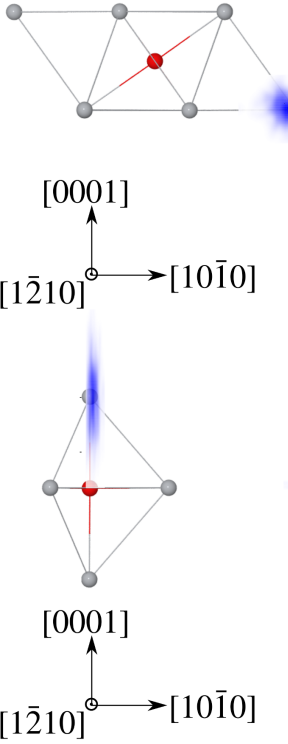

O

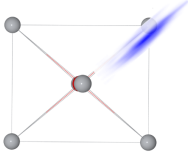

[1̄̄10]

[21̄11]

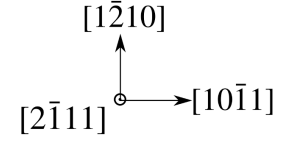

$3 \mathrm{NN}$

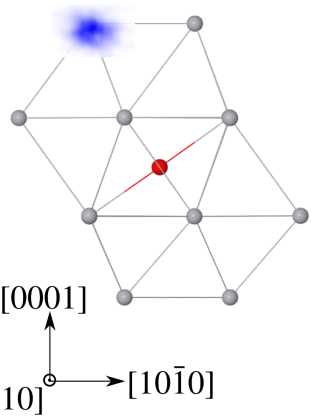

[1̄̄10]
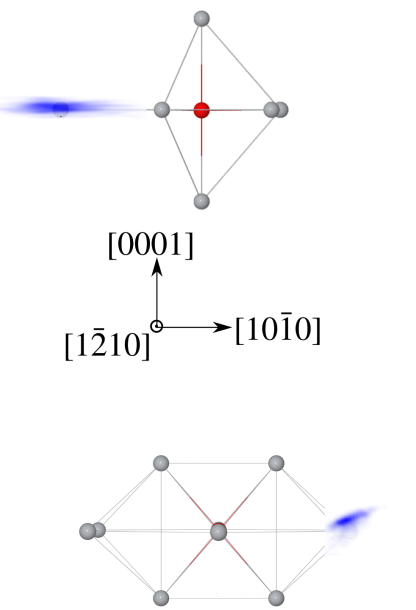

$[2 \overline{1} 11]^{\odot} \longrightarrow[1 \overline{2} 10]$

[10̄11]

Figure 2: Distributions of force errors for the first three nearest neighbor atoms for octahedral (top row), hexahedral (middle row) and crowdion (bottom row) sites. Bayesian sampling produces a distribution of potential parameters, and those parameters then produce a distribution of predictions. The $\mathrm{O}$ atom is red and Ti atoms are gray. The left column of geometries shows the positions of the first three nearest neighbor atoms of the three interstitial sites. The three subsequent columns present the force error distributions on the first nearest-neighbor $(\mathrm{NN})$, the second $\mathrm{NN}$ and the third NN. The forces are oriented such that the force error component perpendicular to the plane of the page is zero due to symmetry. The blue distributions are spatial histograms of the force error vectors starting from the corresponding atoms; each single force represents one potential parameter from the likelihood sample. The force errors span along the Ti-O direction except for the second and the third nearest neighbor ones of octahedral site. The forces are scaled such that $1 \mathrm{eV} / \AA$ appears as $2 \AA$ (roughly the oxygen-titanium distance). The magnitude of the force errors decreases as the Ti-O distance increases for the same interstitial geometry, and the octahedral and hexahedral force errors are smaller than the crowdion ones. 
Table 3: Comparison of GA potential predictions for $\mathrm{O}$ interstitial site energies (eV) and transition barriers (eV) to DFT calculations [7] and analytic MEAM predictions [14]. The initial geometries for interstitial site relaxations are the DFT relaxed geometries. The NEB calculations for the transition barriers use the potential relaxed geometries as the initial and final configurations. The DFT data is from Wu and Trinkle. 77 The analytic MEAM predictions are from Joost et al. [14] The potential predictions are calculated using the parameters in Table 2 with LAMMPS. The mean and standard deviation of the interstitial energy levels predictions are in the parentheses, which are computed using the potential ensemble. The GA crowdion energy level error is the largest, which also leads to lower barriers coming out from the crowdion site. The GA potential predicts accurate interstitial energy levels for the o-site and h-site, and accurate barriers between $\mathrm{o}-\mathrm{h}$ and o-o.

\begin{tabular}{cccc}
\hline \hline & DFT & MEAM, this study & MEAM \\
\hline$E_{\text {relax h }}-E_{\text {relax o }}$ & 1.19 & $1.099(1.062 \pm 0.147)$ & 1.14 \\
$E_{\text {relax c }}-E_{\text {relax o }}$ & 1.88 & $1.675(1.717 \pm 0.204)$ & 1.97 \\
$E_{\text {oh }}-E_{\mathrm{o}}$ & 2.04 & 1.921 & 1.98 \\
$E_{\mathrm{oc}}-E_{\mathrm{o}}$ & 2.16 & 1.852 & 2.04 \\
$E_{\mathrm{oh}}-E_{\mathrm{h}}$ & 0.85 & 0.822 & 0.84 \\
$E_{\mathrm{hc}}-E_{\mathrm{h}}$ & 0.94 & 0.766 & 0.85 \\
$E_{\mathrm{oc}}-E_{\mathrm{c}}$ & 0.28 & 0.177 & 0.07 \\
$E_{\mathrm{hc}}-E_{\mathrm{c}}$ & 0.24 & 0.190 & 0.02 \\
$E_{\mathrm{oo}}-E_{\mathrm{o}}$ & 3.25 & 3.047 & 2.94 \\
\hline \hline
\end{tabular}

calculations, all the values are obtained using the parameters in Table 2 Our GA potential accurately predicts the o-o barrier and o-h barrier, as expected from the optimal weight analysis: significant force entries weights for the o-site, the h-site, o-h and o-o. The larger energy error for o-h is compensated by the force errors and gives the correct barrier values when relaxed. Similar arguments apply for o-o barrier as well. Although the unrelaxed energy differences for the transition states of o-c and h-c have small errors, the force errors cause the underestimation of the c-site barriers. However, we still improve these barriers compared to the analytic MEAM[14]. With better transition barrier predictions, our potential provides a more accurate description of the diffusion behavior of oxygen in HCP titanium.

Figure 3 shows that the oxygen presence in the $\mathrm{Ti}(10 \overline{1} 0)$ prismatic plane makes 


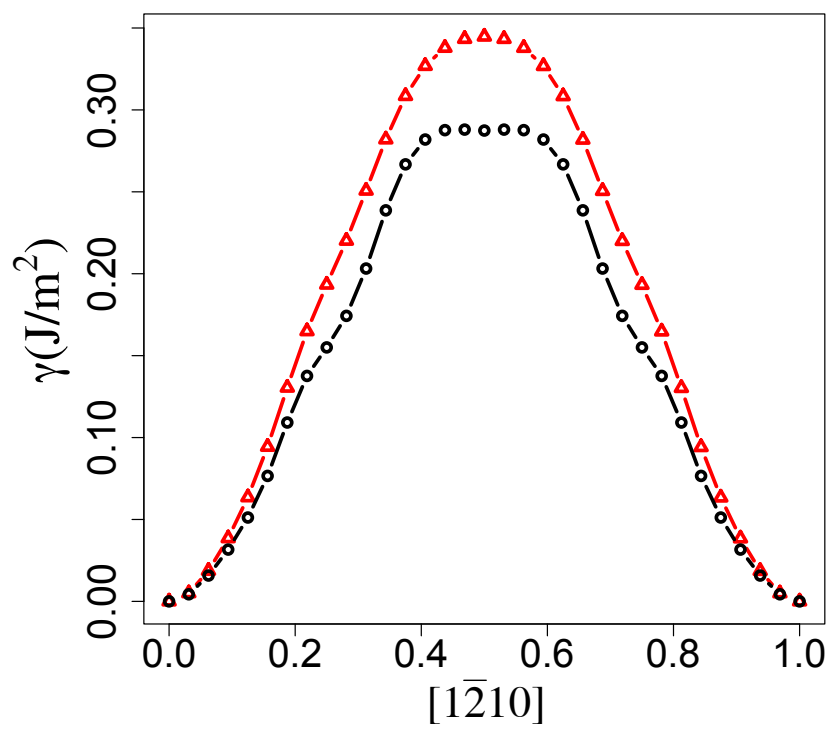

Figure 3: Generalized stacking fault energy of pure Ti (black) and Ti-O (red) in the stacking fault at octahedral site along $[1 \overline{2} 10]$ direction using the GA potential. The pure Ti MEAM stacking fault has a very shallow minimum. The introduction of $\mathrm{O}$ makes the stacking fault unstable. The increase in the stacking fault energy due to $\mathrm{O}$ is $0.050 \mathrm{~J} \cdot \mathrm{m}^{-2}$ at the peak.

the stacking fault along the $[1 \overline{2} 10]$ unstable and increases the generalized stacking fault (GSF) energy, as DFT predicts. We include DFT relaxed stacking fault configurations in the testing set to train the potential with similar atomic environments to a dislocation. However, we do not apply stacking fault relaxation calculations in the testing set. The Ti-MEAM potential predicts a stable prismatic stacking fault in pure Ti with a GSF of $0.293 \mathrm{~J} \cdot \mathrm{m}^{-2}$ with a shallow barrier. The $\mathrm{O}$ atom increases the GSF to $0.343 \mathrm{~J} \cdot \mathrm{m}^{-2}$. Our potential gives good estimates of the increase of the stacking fault energy due to the $\mathrm{O}$ atom: The DFT GSF energy[8] increase is $0.042 \mathrm{~J} \cdot \mathrm{m}^{-2}$ whereas our GA potential gives $0.05 \mathrm{~J} \cdot \mathrm{m}^{-2}$. The interaction of $\mathrm{O}$ with a prismatic stacking fault is a good surrogate for O interaction with the dislocations as both "easy" and "hard" type of prismatic stacking faults are found in the dislocation core.[18]

Figure 4 shows the stable octahedral sites and hexahedral sites near a Ti $\frac{a}{3}[1 \overline{2} 10]$ screw dislocation and the interaction energy of oxygen with the core. Ghazisaeidi and Trinkle found that the Ti spline MEAM potential produces two stable core structures for 
Ti screw dislocations: a mirrored core structure and an unmirrored core structure.[18] We choose to study the $\mathrm{O}$ interaction with the mirrored core, as the mirrored core is a low energy state and the unmirrored core transforms to mirrored core under strain.[18] The GA potential predicts that $\mathrm{O}$ relaxes to either octahedral or hexahedral sites near the dislocation core with some stable tetrahedral sites. We calculate the interaction energy of the $\mathrm{O}$ atom using a reference of $\mathrm{O}$ at an octahedral site far from the dislocation core. The majority of the octahedral site interaction energies are in the range of $\pm 0.1 \mathrm{eV}$. The hexahedral sites have larger interaction energies than the octahedral sites. The lower energy hexahedral sites distribute near the dislocation core, which are more similar to distorted octahedral sites. The tetrahedral sites near the core have an energy in range of $0.7 \pm 0.1 \mathrm{eV}$, although the tetrahedral site is not stable in bulk Ti. The hexahedral sites that are further away from the core have interaction energies close to the bulk energy difference of $1.19 \mathrm{eV}$. Therefore, we conclude that the interaction of $\mathrm{O}$ with the Ti dislocation core is weak. We look into three differential displacement (DD) maps of octahedral sites with interaction energies $0.148 \mathrm{eV}, 0.109 \mathrm{eV}$ and $-0.232 \mathrm{eV}$ to understand the local change in Ti screw dislocation core under stronger $\mathrm{O}$ interactions.

Figure 5 shows the change of the $\mathrm{Ti}$ screw dislocation core structure for $\mathrm{O}$ atom at three octahedral sites near the dislocation core. The oxygen atom in the left figure is placed at the center of the core with a strong repulsive energy of $0.148 \mathrm{eV}$. The DD map shows little changes in the dislocation core geometry induced by the oxygen atom. The strong repulsive energy is consistent with the experiment findings, which suggests that oxygen would act as a solid solution strengthener [2, 3] and the repulsive interaction between oxygen and the dislocation contributes to the strength increase[4]. The oxygen atom in the middle figure is placed neighboring the dislocation core in the basal plane (not the primary slip plane), the interaction energy is lower but still repulsive. The DD map shows the oxygen creating distortions in the dislocation core geometry while the core remains mirrored. The oxygen atom in the right figure is placed in the same slip plane as the dislocation with an attractive interaction energy. The DD map shows that the mirrored dislocation core geometry has transformed to an unmirrored core structure, which is a higher energy dislocation core for Ti without oxygen. Further DFT or experimental evidence is required to verify the predictions of 


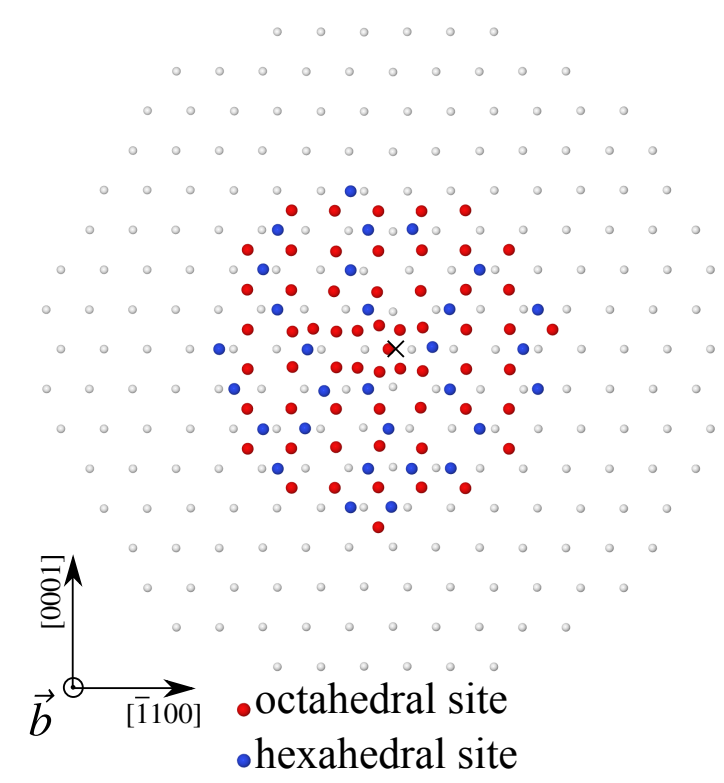

(a)

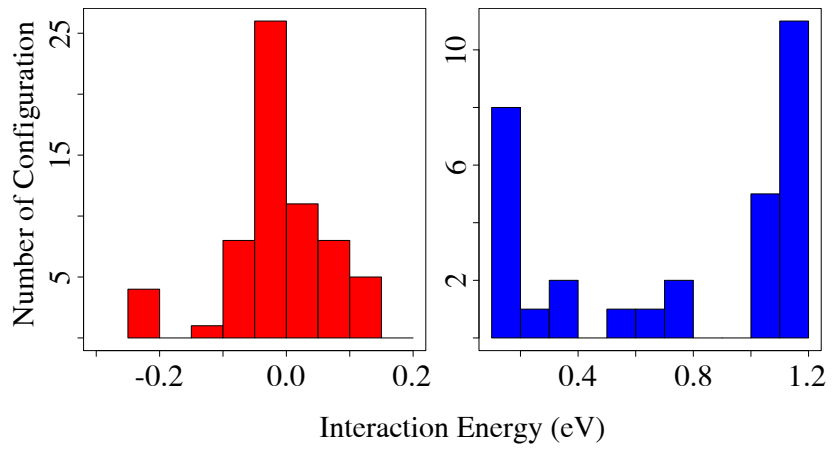

(b)

Figure 4: Interaction of $\mathrm{O}$ with a $a / 3[1 \overline{2} 10]$ screw dislocation in Ti. The dislocation core is marked by an $\times$. We put the $\mathrm{O}$ atoms at octahedral, hexahedral and crowdion sites and relax the geometry. (a) Relaxed positions of $\mathrm{O}$ atoms near the $\mathrm{Ti}$ screw dislocation core. The majority of the octahedral site remains at octahedral site (red atoms), while hexahedral and crowdion sites near the dislocation core displace out of the basal plane into tetrahedral sites (blue atoms) if they remain stable at all. The tetrahedral site is not stable in bulk $\mathrm{HCP} \mathrm{Ti}$, or far from the dislocation core. (b) Histogram of $\mathrm{O}$ interaction energies with a Ti screw dislocation. The corresponding colors represent the energy distributions of the starting interstitial site types. The octahedral-sites and distorted octahedral sites near the dislocation core have interaction energies less than $0.4 \mathrm{eV}$. The tetrahedral sites have interaction energies in range $(0.5-0.8 \mathrm{eV})$. The hexahedral sites have the interaction energy close to the difference between octahedral and hexahedral sites in bulk, $1.19 \mathrm{eV}$. 


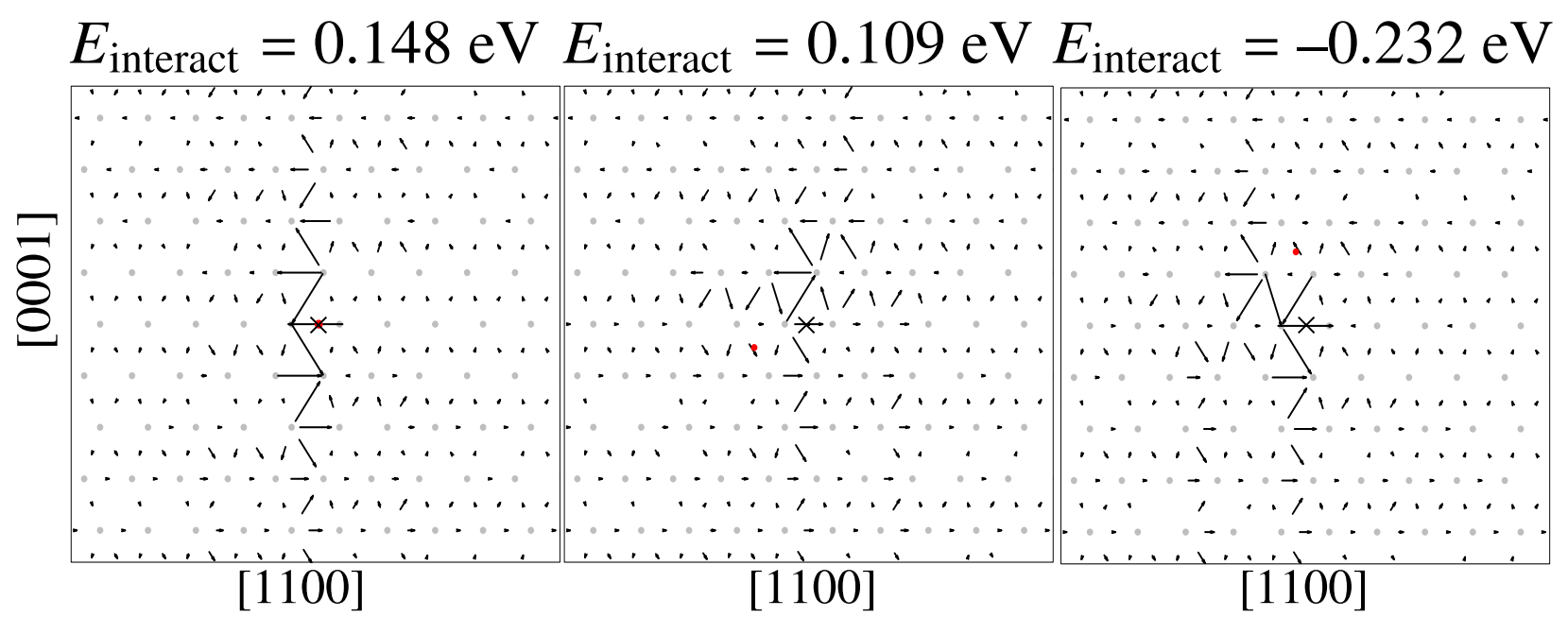

Figure 5: Differential displacement (DD) maps for O near a Ti screw dislocation. Gray atoms are Ti and the $\mathrm{O}$ atom is red; the dislocation core is marked with $\times$. The oxygen atom in the left figure is close to the dislocation core, and maintains a mirrored dislocation core. The middle figure shows significant changes to the dislocation core in the neighborhood of the oxygen atom, while the dislocation core remains mirrored. The right figure, shows that the transformation of dislocation core geometry to an unmirrored core lead to an attractive interaction energy.

the potential for $\mathrm{O}$ near Ti dislocations.

\section{Conclusion}

We develop an empirical potential that accurately models the Ti-O interaction for oxygen in the dilute limit. The potential is one of the first binary MEAM functional form developed using the database optimization algorithm[20] to automate the potential fitting process. We use the logistic function to assess potential transferability. A parallel genetic algorithm finds the optimal Ti-O fitting database and potential parameters. We directly test the potential's transferability against a variety of properties such as $\mathrm{O}$ interstitial energy levels, transition barriers between the $\mathrm{O}$ interstitial sites and $\mathrm{O}$ in the Ti prismatic stacking fault. The empirical potential model is able to reproduce $\mathrm{O}$ interactions with $\mathrm{Ti}$ in various atomic environments corresponding to different crystalline defects. We find weak and short-ranged $\mathrm{O}$ interaction with a Ti screw dislocation using our potential model. We expect further DFT studies can verify this result. 
The potential is also applicable to molecular dynamics simulations of $\mathrm{O}$ diffusion in pure Ti or near Ti defects.

\section{Acknowledgements}

The authors thank Michael R. Fellinger, Ravi Agarwal and Zebo Li for useful comments and discussions. Computational resources were provided on the Taub and Golub clusters at University of Illinois, Urbana-Champaign. The research was supported by the Office of Naval Research through ONR Award No. N000141210752. The potential parameters are available through the NIST Potential Repository; see Ref. [32]. The fitting database, testing set, and raw VASP input and output files are archived by NIST at materialsdata.nist.gov; see Ref. [27].

\section{References}

[1] Lütjering G, Williams J. Titanium. Berlin: Springer; 2003.

[2] Tyson W. Solution hardening of titanium by oxygen. Scripta Metallurgica 1969;3(12):917 -21. URL: http://www.sciencedirect.com/ science/article/pii/0036974869902415, doi/http://dx.doi. org/10.1016/0036-9748(69)90241-5

[3] Conrad H. Effect of interstitial solutes on the strength and ductility of titanium. Progress in Materials Science 1981;26(2âa“4):123 - 403. URL: http://www.sciencedirect.com/science/article/ pii/0079642581900013. doi/http://dx.doi.org/10.1016/ 0079-6425(81)90001-3.

[4] Yu Q, Qi L, Tsuru T, Traylor R, Rugg D, Morris JW, et al. Origin of dramatic oxygen solute strengthening effect in titanium. Science 2015;347(6222):635-

9. URL: http://www.sciencemag.org/content/347/6222/635. abstract doi:10.1126/science.1260485. 
[5] Oberson PG, Ankem S. Why twins do not grow at the speed of sound all the time. Phys Rev Lett 2005;95:165501. URL: http: //link.aps.org/doi/10.1103/PhysRevLett.95.165501. doi:10.1103/PhysRevLett.95.165501.

[6] Oberson P, Wyatt Z, Ankem S. Modeling interstitial diffusion controlled twinning in alpha titanium during low-temperature creep. Scripta Materialia 2011;65(7):638 -41. URL: http://wWw.sciencedirect. com/science/article/pii/S1359646211003885 doi:http: //dx.doi.org/10.1016/j.scriptamat.2011.06.049

[7] Wu HH, Trinkle DR. Direct diffusion through interpenetrating networks: Oxygen in titanium. Phys Rev Lett 2011;107:045504. URL: http://link . aps.org/doi/10.1103/PhysRevLett.107.045504, doi:10.1103/ PhysRevLett.107.045504.

[8] Ghazisaeidi M, Trinkle D. Interaction of oxygen interstitials with lattice faults in ti. Acta Materialia 2014;76:82 -6. URL: http: / / www. sciencedirect. com/science/article/pii/S1359645414003735 doi.http: //dx.doi.org/10.1016/j.actamat.2014.05.025.

[9] Kwasniak P, Muzyk M, Garbacz H, Kurzydlowski K. Influence of c, h, $\mathrm{n}$, and o interstitial atoms on deformation mechanism in titanium-first principles calculations of generalized stacking fault energy. Materials Letters 2013;94:92 -4. URL: http://wWw.sciencedirect.com/science/ article/pii/S0167577X12017181 doi:http://dx.doi.org/10. $1016 / j . m a t l e t .2012 .12 .002$.

[10] Baskes MI. Application of the embedded-atom method to covalent materials: A semiempirical potential for silicon. Phys Rev Lett 1987;59:26669. URL: http://link.aps.org/doi/10.1103/PhysRevLett.59. 2666 doi:10.1103/PhysRevLett.59.2666

[11] Baskes MI, Nelson JS, Wright AF. Semiempirical modified embeddedatom potentials for silicon and germanium. Phys Rev B 1989;40:6085- 
100. URL: http://link.aps.org/doi/10.1103/PhysRevB.40. 6085 doi:10.1103/PhysRevB.40.6085

[12] Daw MS, Baskes MI. Semiempirical, quantum mechanical calculation of hydrogen embrittlement in metals. Phys Rev Lett 1983;50:12858. URL: http://link.aps.org/doi/10.1103/PhysRevLett.50. 1285 doi:10.1103/PhysRevLett.50.1285.

[13] Daw MS, Baskes MI. Embedded-atom method: Derivation and application to impurities, surfaces, and other defects in metals. Phys Rev B 1984;29:644353. URL: http://link.aps.org/doi/10.1103/PhysRevB.29. 6443. doi:10.1103/PhysRevB.29.6443.

[14] Joost WJ, Ankem S, Kuklja MM. A modified embedded atom method potential for the titanium-oxygen system. Modelling and Simulation in Materials Science and Engineering 2015;23(1):015006. URL: http: / / stacks . iop. org/0965-0393/23/i=1/a=015006.

[15] Lenosky TJ, Sadigh B, Alonso E, Bulatov VV, de la Rubia TD, Kim J, et al. Highly optimized empirical potential model of silicon. Modell Simul Mater Sci Eng 2000;8(6):825. URL: http://stacks.iop.org/0965-0393/8/i= $6 / \mathrm{a}=305$

[16] Hennig RG, Lenosky TJ, Trinkle DR, Rudin SP, Wilkins JW. Classical potential describes martensitic phase transformations between the $\alpha, \beta$, and $\omega$ titanium phases. Phys Rev B 2008;78:054121. URL: http:// link.aps.org/ doi/10.1103/PhysRevB.78.054121, doi:10.1103/PhysRevB.78. 054121

[17] Park H, Fellinger MR, Lenosky TJ, Tipton WW, Trinkle DR, Rudin SP, et al. $A b$ initio based empirical potential used to study the mechanical properties of molybdenum. Phys Rev B 2012;85:214121. URL: http: / / link . aps.org/ doi/10.1103/PhysRevB.85.214121, doi:10.1103/PhysRevB.85. 214121 
[18] Ghazisaeidi M, Trinkle D. Core structure of a screw dislocation in ti from density functional theory and classical potentials. Acta Materialia 2012;60(3):1287 -92. URL: http://www.sciencedirect. com/science/article/pii/S1359645411008147 doi/http: //dx.doi.org/10.1016/j.actamat.2011.11.024.

[19] Ercolessi F, Adams JB. Interatomic potentials from first-principles calculations: The force-matching method. Europhys Lett 1994;26(8):583. URL: http:// stacks.iop.org/0295-5075/26/i=8/a=005.

[20] Zhang P, Trinkle DR. Database optimization for empirical interatomic potential models. Modelling Simul Mater Sci Eng 2015;23(6):065011. URL: http:// stacks.iop.org/0965-0393/23/i=6/a=065011.

[21] Plimpton S. Fast parallel algorithms for short-range molecular dynamics. Journal of Computational Physics 1995;117(1):1 - 19. URL: http://lammps. sandia.gov doi:http://dx.doi.org/10.1006/jcph.1995.1039.

[22] Frederiksen SL, Jacobsen KW, Brown KS, Sethna JP. Bayesian ensemble approach to error estimation of interatomic potentials. Phys Rev Lett 2004;93:165501. URL: http://link.aps.org/doi/10.1103/ PhysRevLett.93.165501, doi $10.1103 /$ PhysRevLett.93.165501.

[23] Congdon P. Bayesian Statistical Modelling. 2nd edition ed.; Wiley; 2007.

[24] Mitchell M. An Introduction to Genetic Algorithms. Cambridge, MA, USA: MIT Press; 1996. ISBN 0-262-13316-4.

[25] Kresse G, Hafner J. Ab initio molecular dynamics for liquid metals. Phys Rev B 1993;47:558-61. URL: http://link.aps.org/doi/10.1103/ PhysRevB.47.558, doi:10.1103/PhysRevB.47.558

[26] Kresse G, Furthmüller J. Efficient iterative schemes for $a b$ initio total-energy calculations using a plane-wave basis set. Phys Rev B 1996;54:11169-86. URL: http://link.aps.org/doi/10.1103/PhysRevB.54.11169. doi:10.1103/PhysRevB.54.11169. 
[27] Trinkle DR, Zhang P. Data citation: Fitting database entries for a modified embedded atom method potential for interstitial oxygen in titanium. 2016. Http://hdl.handle.net/11256/782.

[28] Henkelman G, Uberuaga BP, Jónsson H. A climbing image nudged elastic band method for finding saddle points and minimum energy paths. J Chem Phys 2000;113:9901-4.

[29] Vanderbilt D. Soft self-consistent pseudopotentials in a generalized eigenvalue formalism. Phys Rev B 1990;41(11):7892-5.

[30] Kresse G, Hafner J. Norm-conserving and ultrasoft pseudopotentials for first-row and transition elements. Journal of Physics: Condensed Matter 1994;6(40):8245. URL: http://stacks.iop.org/0953-8984/6/i=40/a=015.

[31] Hennig RG, Trinkle DR, Bouchet J, Srinivasan SG, Albers RC, Wilkins JW. Impurities block the $\alpha$ to $\omega$ martensitic transformation in titanium. Nat Mater 2005;4(2):129-33. URL: http://dx.doi.org/10.1038/nmat1292

[32] Becker CA, Tavazza F, Trautt ZT, Buarque de Macedo RA. Considerations for choosing and using force fields and interatomic potentials in materials science and engineering. Current Opinion in Solid State and Materials Science 2013;17:277-83. URL: http://www.ctcms.nist.gov/potentials. doi:10.1016/j.cossms.2013.10.001. 

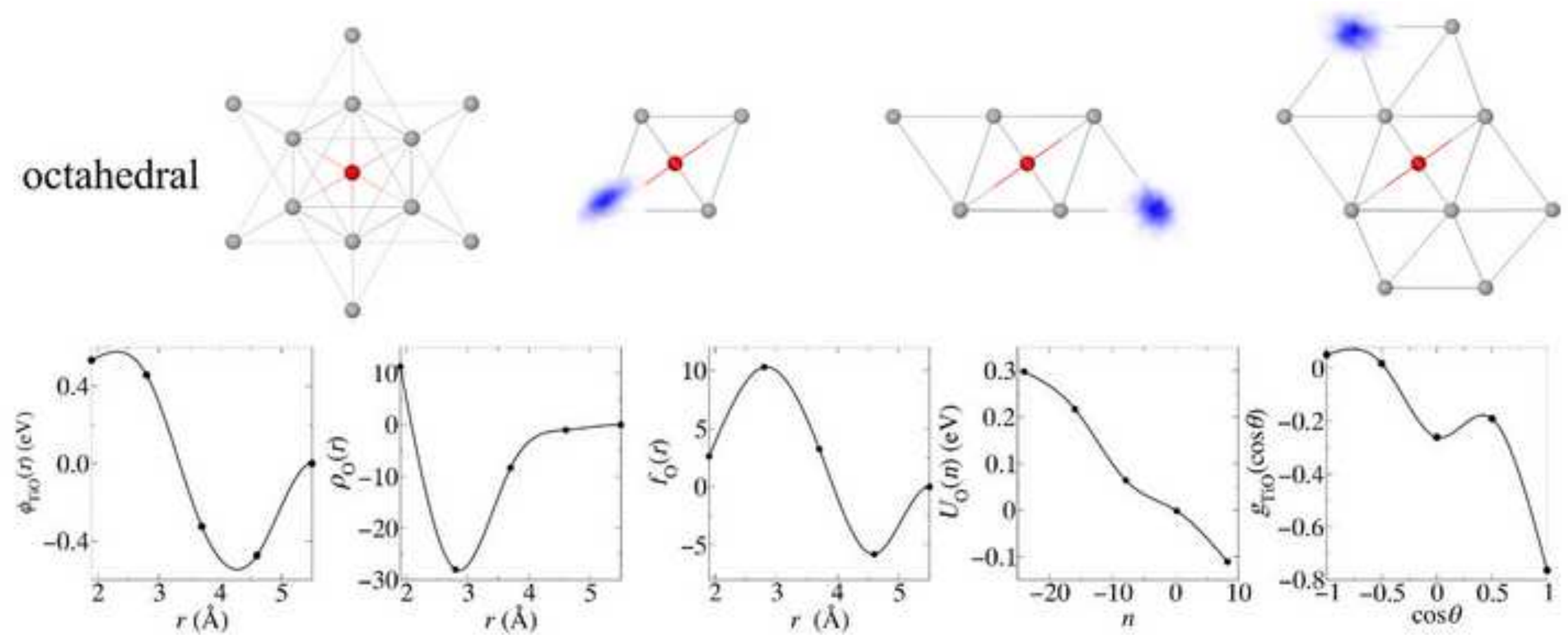